\title{
Ex Vivo Open Reconstruction of Hilar Renal Artery Aneurysms: A Single-Center Experience
}

\author{
Mirko Menegolo, Elda Chiara Colacchio, \\ Lucrezia Furian, Paolo Rigotti, \\ Jacopo Taglialavoro, Michele Piazza, \\ Michele Antonello, and Franco Grego
}

\subsection{Epidemiology and Guidelines}

The renal artery aneurysms (RAAs) represent 3\% of all visceral aneurysms. Their incidence rate is about $0.1 \%$ in the general population [1], even if angiographic findings report a little higher incidence $(0.3-2.5 \%)$ [2] with a modest prevalence in female patients.

RAAS are mainly detected in the sixthseventh decade, and female patients are associated with an earlier incidence [3]. True RAAs are extra-parenchymal in $90 \%$ of cases, while intraparenchymal RAAs represent $10 \%$ of cases, and they are often multiple and congenital. RAAs can be saccular in $75 \%$ of cases or fusiform. Saccular RAAs usually affect the main renal artery bifurcation, while fusiform aneurysms often involve the main arterial trunk [4].

M. Menegolo $(\bowtie) \cdot$ E. C. Colacchio $\cdot$ J. Taglialavoro

M. Piazza · M. Antonello · F. Grego

Department of Cardiac, Thoracic and Vascular

Sciences, Vascular and Endovascular Surgery,

University of Padova, Padova, Italy

e-mail: mirko.menegolo@unipd.it;

michele.antonello.1@unipd.it;

franco.grego@unipd.it

L. Furian · P. Rigotti

Department of Surgery, Oncology and

Gastroenterology, Surgery and Organ Transplantation

Unit, University of Padova, Padova, Italy

e-mail: lucrezia.furian@unipd.it;

paolo.rigotti@unipd.it

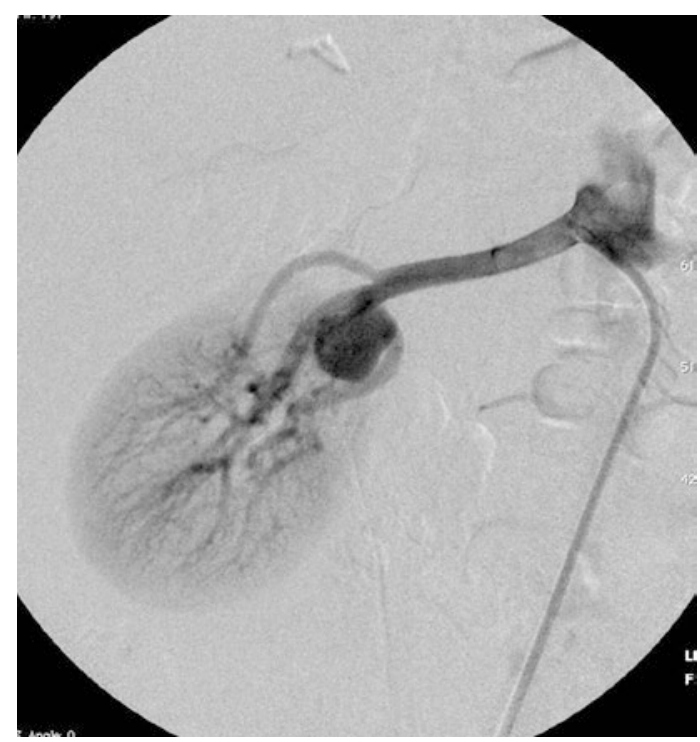

Fig. 12.1 Hilar renal artery aneurysm. The digital subtraction angiography shows the distal location of the renal aneurysm involving the origin of the terminal branches

RAAs can be bilateral (10-20\%) or associated with a multiple location. High calcifications are detected in $18-68 \%$ of cases [1].

HRAAs represent a rare subgroup of RAAs, located in the distal part of the renal artery, very close to the renal hilum even though they are considered extra-parenchymal (Fig. 12.1).

In addition to "true aneurysms," the main renal arteries and their collaterals can be affected by: 
- Pseudoaneurysms, which can occur after trauma or invasive procedures, such as renal biopsy, nephrostomy, or selective catheterization of renal arteries.

- Arteriovenous malformations, congenital or posttraumatic, that can evolve in dangerous dilatative diseases.

- Aneurysms following artery dissection.

- Mycotic aneurysms.

- Aneurysms secondary to pathologies, such as panarteritis nodosa, tuberculosis, neurofibromatosis, and Kawasaki disease; they are generally intraparenchymal and lead to nephrectomy.

RAAs have a slow growth rate $(0.06-0.6 \mathrm{~mm} /$ year) [1] with no difference in terms of aneurysm morphology or calcification. The rupture rate is estimated at 3-5\% with a non-gestational mortality $<10 \%$ [1]. For those reasons, the surgical indications are still controversial. Actually, indications for RAA treatment include diameters of more than $2 \mathrm{~cm}$, aneurysms of any size in females within childbearing age, symptoms such as pain, medical refractory hypertension, thromboembolism, concomitant symptomatic dissection, and rupture $[1,5,6]$.

\subsection{Clinical Presentation}

Most RAAs are asymptomatic, and before the routine use of computed tomography angiography (CTA), their incidence was much lower since they were mainly detected during autopsy.

Currently, RAAs are often discovered during instrumental examinations for other pathologies or during investigation for medically refractory hypertension.

Hypertension seems to be the most common symptom, in approximately $70-100 \%$ of patients [1], and it is still unclear whether the presence of the aneurysm causes hypertension. Many theories have been advanced to explain this association:

- Hemodynamic changes from turbulent blood flow within the aneurysm resulting in decreased distal renal artery perfusion [7]
- Distal parenchymal embolization causing microvascular ischemia with a consequent releasing of angiotensin II [8]

- Compression or kinking of renal artery branches [1]

- Coexistent renal artery steno-occlusive disease that has been identified in $7-66 \%$ of patients with RAA [1]

Most authors report a significant reduction in arterial blood pressure in the postoperative period, both after surgical and endovascular correction [9].

Other symptoms include acute flank pain and hematuria, especially if the aneurysm is associated with dissection or hydronephrosis, in cases of large intrarenal RAAs [4].

RAAs may manifest with clinical signs of intra-abdominal arterial rupture and therefore must be treated as a vascular surgical emergency.

The highest rupture rate occurs in saccular aneurysms and during pregnancy with a maternal/ gestational mortality of $80 \%$ [10].

\subsection{Diagnosis}

The patient's history and the medical examination are generally silent, except for large aneurysms in very lean patients. The diagnosis of RAAs often occurs by chance, especially during investigation for other diseases.

The first-level examination in the diagnosis of a RAA is ultrasound (US). It should be performed by experienced staff, and it gives morphological and flow information that are important both in the preoperative period (to exclude associated steno-occlusive diseases) and in the follow-up, in order to evaluate arterial patency and kidney perfusion.

The US technique has the advantage of being inexpensive and noninvasive. However, it is operator-dependent, and favorable anatomical conditions are needed (lean patient, localization of the aneurysm, poor intestinal meteorism) [11].

Computed tomography angiography (CTA) is a second-level exam, and it is essential both for diagnosis and for postoperative follow-up. It is a 


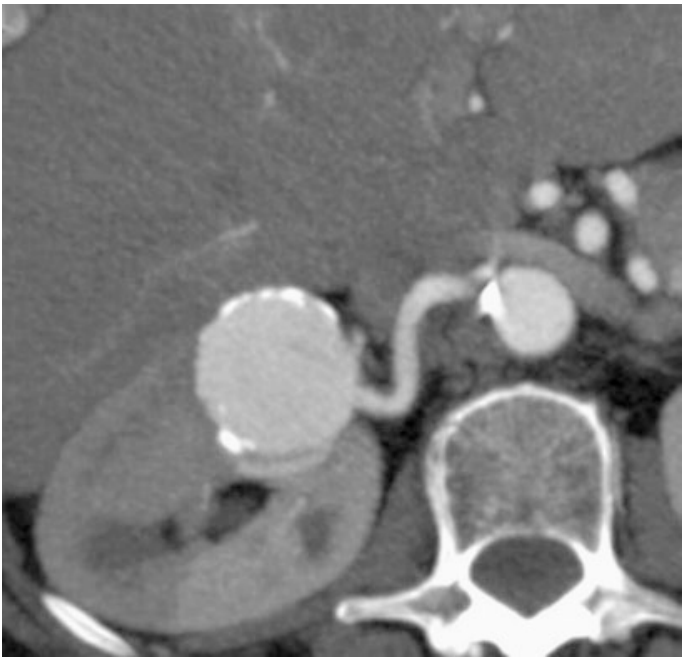

Fig. 12.2 The computed tomography allows in the majority of cases the perfect anatomical definition of renal artery aneurysm even if located closely to the kidney parenchyma

low-invasive technique that accurately identifies RAAs and allows for adequate anatomical definition and precise preoperative planning. CTA is as accurate as conventional angiography, with the advantage of being faster, cheaper, and less invasive. CTA should be the first choice in evaluating an RAA [12] (Fig. 12.2).

Magnetic resonance angiography (MRA) is a diagnostic exam similar to CTA. It provides information about the location of RAA and the vascular anatomy. It is superimposable to angiographic findings in $93 \%$ of cases [13].

However, CTA gives a better spatial 3D resolution than MRA. Moreover, MRA does not provide detailed information about the vessel wall [14].

In this regard, while CTA is preferable for preoperative planning, MRA can be used for followup avoiding X-ray exposure and nephrotoxicity of contrast medium.

Digital subtraction angiography (DSA) is still the diagnostic tool of choice especially in cases involving renal artery bifurcations or when the morphology of the aneurysm is not easy to analyze with CTA (Fig. 12.3). It provides the most accurate images of arterial flow, precise location of the aneurysm, and vascularization of the organ [14]. However, this examination must

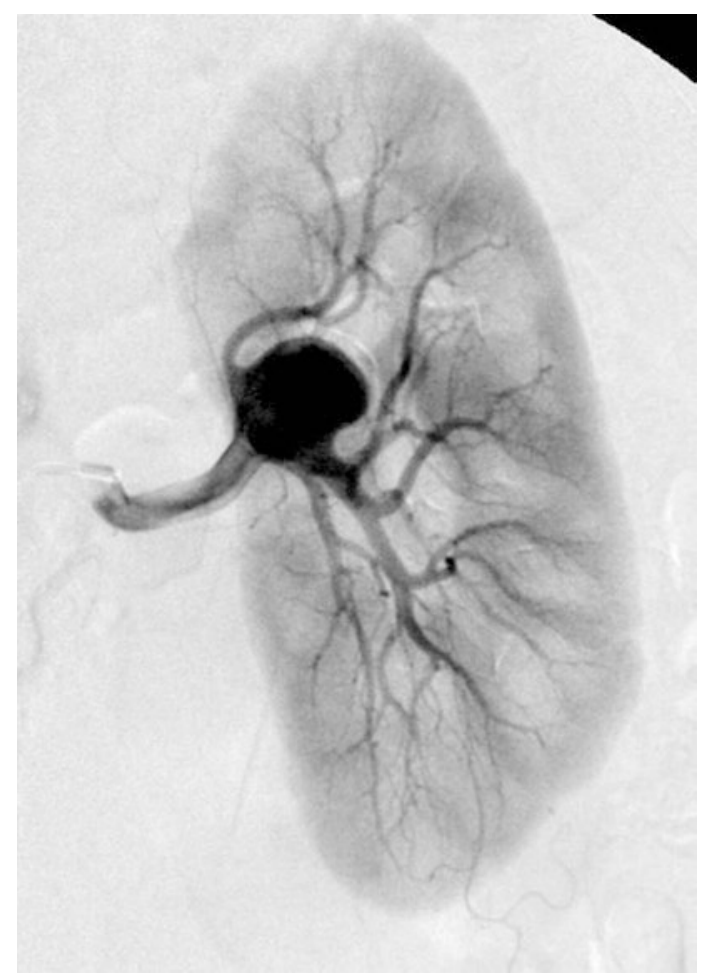

Fig. 12.3 Digital subtraction angiography of a hilar renal artery aneurysm involving collateral branches

be considered not only a diagnostic tool, but it must be aligned with the surgical procedure.

Despite its invasiveness, the risk associated with arterial puncture, use of contrast medium, and intra-arterial devices (catheters, guidewires, etc.) is very low [14].

\subsection{Treatment}

The patient history provides key information on the surgical indication and the best treatment solution. It is important to know the age, sex, fertility (for women), symptoms (e.g., hypertension), and comorbidities that could influence the surgical choice (kidney failure, drug or iodine contrast allergies, heart diseases, coagulation disorders, previous abdominal surgery, or medical therapy).

Treatment of RAAs is still controversial and several techniques have been proposed over the years. 
Given the difficulties associated with the treatment and the usual slow growth of RAA, some authors have proposed a conservative treatment and to monitor patients by ultrasonography, reserving surgery only for selected cases $[1,15]$. However, in the majority of cases, a treatment solution should be identified.

The best treatment must be decided considering anatomical features, surgeon's ability, and availability of devices and materials.

For several years, open repair (OR) has been the only treatment available, despite the difficulties associated with this very peculiar district.

The endovascular approach has become popular, given its low invasiveness. It includes two main techniques: coil embolization and/or exclusion of the aneurysm by stent positioning [16]. Patients must be selected considering the anatomy of the vessels. However, in expert hands, even cases with a complex renal artery anatomy can be treated if the right devices are available (Fig. 12.4) [17].

HRAAs were classically excluded for both ER and OR and often candidate to nephrectomy, because of their very distal location close to the renal parenchyma. Even nowadays, in the endovascular era, the majority of HRAAs results

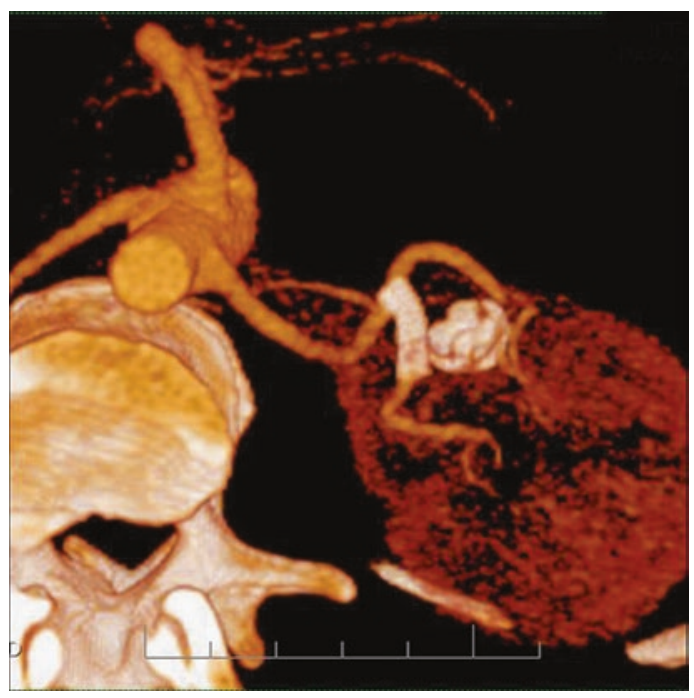

Fig. 12.4 Post-procedural computed tomography of a collateral branch renal artery aneurysm successfully excluded by a covered stent that are unsuitable for an endo-approach because of the difficulty to be super-selective in small vessels. For the same reasons, the conventional "in vivo" open surgical approach could not be feasible either.

"Ex vivo" repair with backbench reconstruction and kidney autotransplantation may facilitate the surgical reconstruction despite the high invasiveness of this approach. In this regard, a total laparoscopic nephrectomy can reduce the surgical impact: the subsequent backbench vascular repair provides many advantages both for the surgeon and for the patient. Kidney autotransplantation can finally be carried out through an extraperitoneal approach in the iliac fossa.

This technique, or the hand-assisted laparoscopic approach, has already been described in small series $[5,18]$.

\subsection{Our Experience}

From September 2011 to December 2017, we treated five patients affected by hilar RAAs using the "ex vivo technique" via a total or handassisted laparoscopic approach and kidney autotransplantation.

Mean age was $60 \pm 4$ years, and two were male patients. Table 12.1 summarizes clinical and demographic characteristics.

The indication for treatment was the diameter of the aneurysm $>2 \mathrm{~cm}$ (mean, $2.9 \pm 0.74 \mathrm{~cm}$ ).

Surgical treatment was performed along with general surgeons, who carried out the nephrectomy and subsequent autotransplantation in all cases.

Table 12.1 Patients demographics, aneurysms characteristics, and arterial reconstructions

\begin{tabular}{l|l}
\hline Variables & $N(\%)$ or mean \pm SD \\
\hline Sex (males) & $2(40)$ \\
\hline Age & $60 \pm 4$ \\
\hline Aneurysm diameter $(\mathrm{cm})$ & $2,9 \pm 0,74$ \\
\hline Aneurysm side (left) & $3(60)$ \\
\hline $\begin{array}{l}\text { Arterial reconstruction } \\
\begin{array}{l}\text { Aneurysmectomy + GSV } \\
\text { patch }\end{array}\end{array}$ & 2 \\
\hline Aneurysmorrhaphy & 3 \\
\hline
\end{tabular}

$S D$ standard deviation, $G S V$ great saphenous vein 


\subsubsection{Surgical Technique}

The patient was placed in a lateral decubitus position, with the hips rotated behind and the arms extended above the head.

This position allowed a rapid surgical conversion with a median laparotomy in case it was needed. The pneumoperitoneum was obtained through a Hasson "open technique" in all cases, with a small paramedian abdominal incision, just above the umbilicus (a "closed technique" with a Veress needle via umbilical access, or left iliac fossa is a superimposable approach).

After the total laparoscopic kidney dissection (releasing it from the adrenal gland and from the peripheral adipose tissue, paying attention to not injure the spleen, the tail of the pancreas, and the adrenal gland), the ureter was cut as far as possible without damaging its vascularization.

Endovenous $5000 \mathrm{IU}$ of heparin and $20 \mathrm{mg}$ of furosemide were administered before cutting the kidney vessels with a laparoscopic stapler (Endo GIA - Covidien).

The kidney was finally extracted by an EndoCatch bag introduced through a Pfannenstiel incision and then perfused with a cold renal preservation Celsior solution [19].

In our series, two patients were treated for a right HRAA, and in these cases, a hand-assisted laparoscopic approach was adopted.

The backbench surgery was performed by vascular surgeons inside a Celsior solution [19].

Aneurysmorrhaphy was performed in three cases, while aneurysm resection with autologous saphenous vein angioplasty was the choice in the other two patients (Fig. 12.5).

At the end of the procedure, the kidney was placed in the right iliac fossa, using a Gibson incision (Fig. 12.6).

Arterial and venous anastomoses were performed in a termino-terminal fashion between the renal artery and the hypogastric artery and between the renal vein and the external iliac vein in all cases. The anastomosis between the ureter and the bladder was performed according to the technique of Lich-Gregoir, which consists in the reimplantation of the ureter on the anterolateral wall of the bladder with an

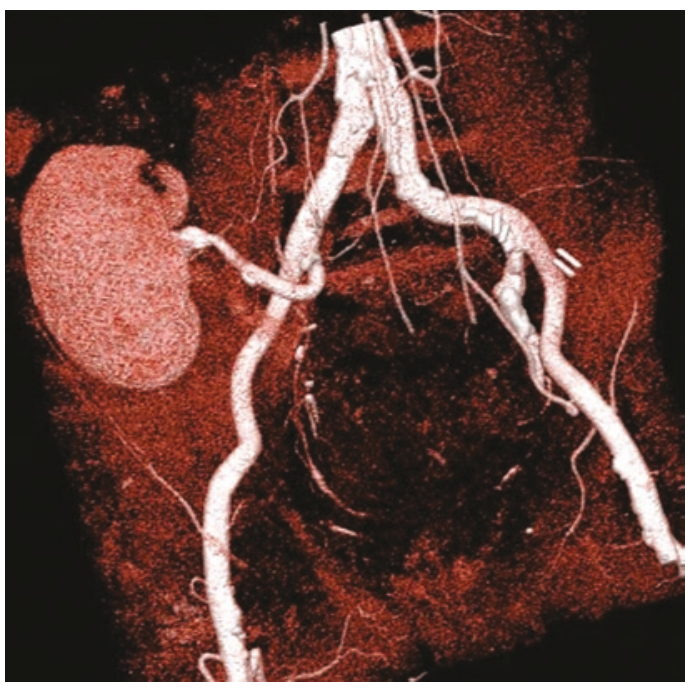

Fig. 12.6 Postoperative computed tomography showing a well-perfused reimplanted kidney in the iliac fossa
Fig. 12.5 Ex vivo bench surgery of a hilar renal artery aneurysm. The removal of a high calcified aneurismal wall (a) allows the reconstruction of the renal artery (b)
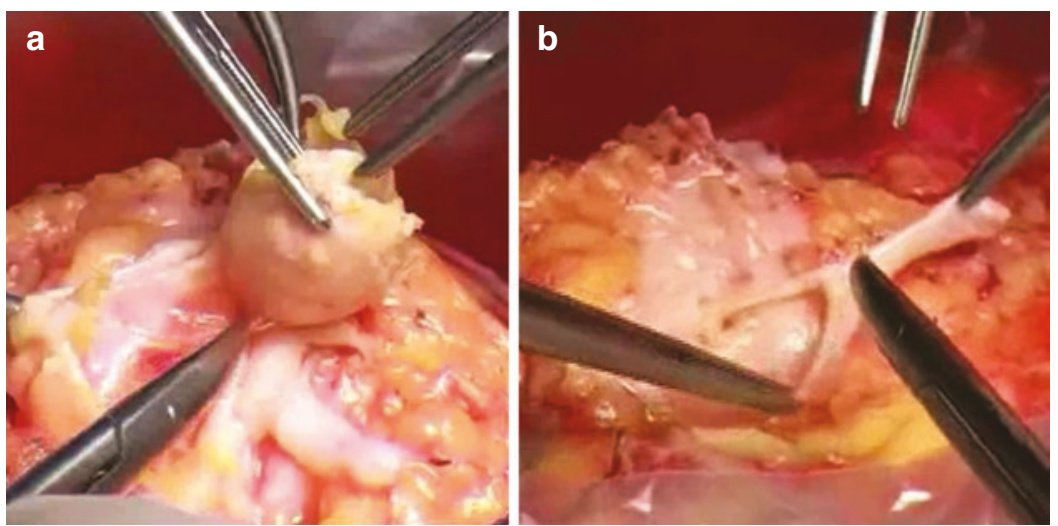
anti-reflux plastic, after positioning a double-J catheter.

A sequential renal perfusion scintigraphy was performed in all patients postoperatively before discharge from hospital.

The follow-up of all patients was achieved by CTA at 1 month and then by US at 6 and 12 months and then yearly, along with renal function monitoring.

\subsubsection{Results}

An aneurysm resection with primary closure was performed in three cases and aneurysm resection with patch angioplasty with great saphenous vein in the other two.

Patients were dismissed after performing renal scintigraphy, which documented the correct functioning of the transplanted organ in four cases $(80 \%)$. Acute renal artery thrombosis occurred only in one case and a nephrectomy was necessary.

All patients were discharged from hospital. The medium follow-up was 9 months (2-36 months). No postoperative deaths were recorded. No postoperative complications occurred in three patients. One patient developed hypertension and it was necessary to introduce medical therapy. There was a doubt regarding a stenosis on the arterial anastomosis, but a CTA excluded it.

\subsection{Discussion and Conclusions}

RAAs are uncommon and, between them, HRAAs are even rarer.

They pose a technical challenge, and they are often unsuitable both for OR and ER.

The ex vivo technique is a potentially valid treatment option and an alternative to nephrectomy for HRAAs where endovascular repair and in situ open repair are not feasible.

The first step is the nephrectomy, and in our series a total or hand-assisted laparoscopic technique was adopted in order to reduce the invasiveness of this approach. In this regard, since pneumoperitoneum reduces renal blood flow, it is important to keep the patient wellhydrated, and a topical application of papaverine on the renal artery to prevent vasospasm may be considered [20].

Diuresis should always be stimulated with a bolus administration of mannitol, and a high level of hydration must be maintained.

Many authors perform the total laparoscopic nephrectomy only in the left kidney, with a single renal artery. The right renal vein is usually too short, and this could eventually increase the risk of venous thrombosis. Furthermore, a total laparoscopic technique could become more difficult to perform with multiple renal arteries. However, experiences of a total laparoscopic technique with a right kidney and/or multiple renal arteries are described [21]. In our series, we adopted the handassisted laparoscopic approach for right diseases.

After the nephrectomy, the following step is backbench surgery, which allows the surgeon to choose the best technical solution for the aneurysm repair.

In this series, both aneurysmorrhaphy and aneurysm resection were performed with autologous saphenous vein angioplasty. The vascular surgeon will make a therapeutic decision based on preoperative imaging but especially on the macroscopic findings. It depends on many factors: the anatomy of the aneurysm, the quality of the arterial wall, and the need to preserve as much of the collateral vessels as possible, with the goal of obtaining a safe, effective, and fast solution. Aneurysmectomy with primary anastomosis between inflow and outflow arteries and replacement of the aneurysmatic tract with autologous veins or prosthetic bypasses are other possible technical options.

During backbench repair, some caution should be exercised in the care of the kidney.

Numerous studies have shown that a warm kidney ischemia time longer than 30 min may cause permanent kidney damage [22-25]. It is also necessary to consider some risk factors related to the patient that can reduce tolerance to ischemia, such as age, diabetes mellitus, and hypertension.

In order to prevent renal ischemic damage, it is mandatory to hydrate the patient during surgery 
and administer mannitol (25-50 g) via intravenous infusion 5-10 min before renal artery crossclamping, in order to reduce cellular swelling.

If the ex vivo surgery time exceeds $30 \mathrm{~min}$, in situ kidney hypothermia is recommended in order to minimize the ischemic damage of the organ. Cooling the kidney surface can extend the duration of the ischemia by up to $3 \mathrm{~h}$ without any permanent damage.

The optimal temperature is $10-15{ }^{\circ} \mathrm{C}$ [18]. The safest method for this purpose is to cool the organ with frozen and shredded saline granules.

In our series, kidneys were perfused with a "Celsior solution," which was first created for cardiac transplantation [19]: it is a highconcentrate solution of $\mathrm{Na}+$, mannitol, lactobionic acid (as in the Wisconsin University solution), and histidine (as in Bretschneider's HTK solution) which prevents cellular edema and removes free radicals.

In conclusion, in the endovascular era, open surgery remains a safe and valid option for treatment of RAAAs even if their location is very distal and proximal to the kidney hilum; in these cases, the ex vivo technique could be considered as an alternative to nephrectomy since the ER and in situ OR are not often feasible.

The total laparoscopic approach for nephrectomy allows this approach to be less invasive, and in this regard, the teamwork (collecting different specialists as vascular surgeons, transplant surgeons/urologists) is mandatory in order to achieve the best results in terms of technical success, patency of renal artery reconstructions, and renal function preservation. However, larger series are needed to assess the real effectiveness of this approach.

\section{References}

1. Coleman DM, Stanley JC. Renal artery aneurysms. J Vasc Surg. 2015;62(3):779-85.

2. Henke PK, Cardneau JD, Welling TH 3rd, Upchurch GR Jr, Wakefield TW, Jacobs LA, Proctor SB, Greenfield LJ, Stanley JC. Renal artery aneurysms: a 35-year clinical experience with 252 aneurysms in 168 patients. Ann Surg. 2001;234(4):454-62.

3. Pfeiffer T, Reiher L, Grabitz K, Grunhage B, Hafele $\mathrm{S}$, Voiculescu A, et al. Reconstrucion for renal artery aneurysm: operative techniques and long-term results. J Vasc Surg. 2003;37:293-300.

4. Calligaro KD, Dougherty MJ. Renovascular disease: aneurysms and arteriovenous fistulae. In: Cronenwett JL, Johnston KW, editors. Rutherford's vascular surgery. 8th ed. Philadelphia: Saunders; 2014.

5. Gallagher KA, Phelan MW, Stern T, Bartlett ST. Repair of complex renal artery aneurysms by laparoscopic nephrectomy with ex vivo repair and autotransplantation. J Vasc Surg. 2008;48(6):1408-13.

6. Klausner JQ, Lawrence PF, Harlander-Locke MP, Coleman DM, Stanley JC, Fujimura N, Vascular LowFrequency Disease Consortium. The contemporary management of renal artery aneurysms. J Vasc Surg. 2015;61(4):978-84.

7. Tham G, Ekelund L, Herrlin K, Lindstedt EL, Olin T, Bergentz SE. Renal artery aneurysms. Natural history and prognosis. Ann Surg. 1983;197:348-52.

8. Hupp T, Allenberg JR, Post K, Roeren T, Meier M, Clorius JH. Renal artery aneurysm: surgical indications and results. Eur J Vasc Surg. 1992;6:477-86.

9. Reiher L, Grabitz K, Sandmann W. Reconstruction for renal artery aneurysm and its effect on hypertension. Eur J Vasc Endovasc Surg. 2000;20:454-6.

10. Stanley JC. Natural history of renal artery stenosis and aneurysms. In: Calligaro KD, Dougherty MJ, Dean $\mathrm{RH}$, editors. Modern management of renovascular hypertension and renal salvage. Baltimore: Williams \& Wilkins; 1996. p. 15.

11. Bielsa AA, Rodriguez JP, Castromil RG. Extraparenchymal renal artery aneurysms: is hypertension an indication for revascularization surgery? Ann Vasc Surg. 2002;16:339-44.

12. Dib M, Sedat J, Raffaelli C, Petit I, Robertson WG, Jaeger P. Endovascular treatment of a wide-neck renal artery bifurcation aneurysm. J Vasc Interv Radiol. 2003;14:1461-4.

13. Bui BT, Oliva VL, Leclerc G, Courteau M, Harel C, Plante R, et al. Renal artery aneurysm: treatment with percutaneous placement of a stent-graft. Radiology. 1995;195:181-2.

14. Tateno T, Kubota Y, Sasagawa I, Sawamura T, Nakada T. Successful embolization of a renal artery aneurysm with preservation of renal blood flow. Int Urol Nephrol. 1996;28:283-7.

15. JP MC Jr, Marshall VF, Whitsell JC 2nd. Indications for surgery on renal artery aneurysms. J Urol. 1975;114(2):177-80.

16. Wei X, Sun Y, Wu Y, Li Z, Zhu J, Zhao Z, Feng R, Jing $Z$. Management of wide-based renal artery aneurysms using noncovered stent-assisted coil embolization. J Vasc Surg. 2017;66(3):850-7.

17. Menegolo M, Frigatti P, Antonello M, Grego F. Stent graft exclusion of a renal artery aneurysm at hilum in a case with complex anatomy. Perspect Vasc Surg Endovasc Ther. 2009;21(4):240-3. Epub 2010 May 20

18. Laser A, Flinn WR, Benjamin ME. Ex vivo repair of renal artery aneurysms. J Vasc Surg. 2015;62(3):606-9. 
19. Catena F, Gazzotti F, Amaduzzi A, Fuga G, Montori G, Cucchetti A, Coccolini F, Vallicelli C, Pinna AD. Pulsatile perfusion of kidney allografts with Celsior solution. Transplant Proc. 2010;42(10):3971-2.

20. Sorokin I, Stevens SL, Cadeddu JA. Periarterial papaverine to treat renal artery vasospasm during robot-assisted laparoscopic partial nephrectomy. J Robot Surg. 2017;12(1):189-91.

21. Ham SW, Weaver FA. Ex vivo renal artery reconstruction for complex renal artery disease. J Vasc Surg. 2014;60(1):143-50.

22. Desai MM, Gill IS, Ramani AP, Spaliviero M, Rybicki L, Kaouk JH. The impact of warm ischaemia on renal function after laparoscopic partial nephrectomy. BJU Int. 2005;95(3): 377-83.

23. Zargar H, Akca O, Ramirez D, Brandao LF, Laydner H, Krishnan J, Stein RJ, Kaouk JH. The impact of extended warm ischemia time on late renal function after robotic partial nephrectomy. J Endourol. 2015;29(4):444-8.

24. Novick AC. Renal hypothermia: in vivo and ex vivo. Urol Clin North Am. 1983;10:637-44.

25. Ward JP. Determination of the optimum temperature for regional renal hypothermia during temporary renal ischaemia. Br J Urol. 1975;47:17-24. 